Relations industrielles

Industrial Relations

\title{
Disputes Procedure in Action, by Richard Hyman, London, Warwick Studies in Industrial Relations, Heineman Educational Books Ltd., 1972, 150 pp.
}

\section{Jean Boivin}

Volume 28, numéro 2, 1973

URI : https://id.erudit.org/iderudit/028407ar

DOI : https://doi.org/10.7202/028407ar

Aller au sommaire du numéro

Éditeur(s)

Département des relations industrielles de l'Université Laval

ISSN

0034-379X (imprimé)

1703-8138 (numérique)

Découvrir la revue

Citer ce compte rendu

Boivin, J. (1973). Compte rendu de [Disputes Procedure in Action, by Richard

Hyman, London, Warwick Studies in Industrial Relations, Heineman

Educational Books Ltd., 1972, 150 pp.] Relations industrielles / Industrial

Relations, 28(2), 437-439. https://doi.org/10.7202/028407ar

Tous droits réservés @ Département des relations industrielles de l'Université Laval, 1973
Ce document est protégé par la loi sur le droit d'auteur. L'utilisation des services d’Érudit (y compris la reproduction) est assujettie à sa politique d'utilisation que vous pouvez consulter en ligne.

https://apropos.erudit.org/fr/usagers/politique-dutilisation/ 
Blanc-Jouvan (France); Thilo Ramm (Allemagne) ; Folke Schmidt (Suède) et Gino Guigni (Italie).

Les trois premiers de ces pays connaissent des juridictions du travail bien établies, lesquelles font cependant montre entre elles de particularités intéressantes. L'Italie, qui avait jadis, elle aussi, institué des tribunaux du travail, s'en remet depuis 1942 aux instances civiles, du moins pour ce qui est des conflits individuels, avec assez de succès selon l'auteur, sauf le problème de la lenteur.

L'établisement de juridictions spécialisées du travail - l'expérience de l'un ou l'autre des autres pays en cause le démontre selon le cas - permet un certain éventail des procédés de tierce intervention: ainsi le stade de la conciliation peut précéder l'étape proprement juridictionnelle. Les «juges》 pourront être issus des secteurs en cause, à tout le moins avoir la familiarité voulue avec le contexte du travail. L'on se retrouvera normalement avec une plus ou moins grande pluralité d'institutions en raison de la diversité de nature des conflits : distinction du conflit individuel et du conflit collectif (France, Allemagne, Italie); du conflit de droit et du conflit d'intérêts (que la tradition ignore en Angleterre)... D'autres questions se posent: rôle de la négociation collective (accentué en Suède); adhésion au droit strict ou introduction de l'équité; place de l'arbitrage volontaire; intégration des palliers d'appel (Allemagne, à la différence de la France); étendue et aménagement de la coercition, en particulier la possibilité de réintégration forcée du salarié (à cet égard les droits français et allemand sont, en pratique, dans une situation d'infériorité par rapport à l'arbitrage américain); choix entre l'accès direct du salarié et le contrôle du recours par le syndicat (seule la Suède s'apparente ici au droit nord-américain)...

D'une façon positive, comme le constate le professeur Aaron dans ses propos introductifs, les solutions qui prévalent dans ces différents pays, y sont évidemment le résultat de données historiques, sociales et économiques, souvent propres; des transplantations indues pourraient être suivies de rejets. Ces synthèses scientifiques d'expériences é- trangères seraient néanmoins susceptibles de contribuer à l'examen de prudentes remises en cause. En particulier, l'étude du professeur Blanc-Jouvan, qui avait, à une autre occasion, pénétré brillamment l'ensemble du système des rapports collectifs aux Etats-Unis, réussit cette fois, dans le cas de son propre pays, à présenter, dans un nombre limité de pages, non seulement la structure et le fonctionnement des institutions dont il s'agit, mais également le contexte socio-juridique qui les soustend. Ainsi en est-il notamment des Conseils de prud'hommes, juridiction limitée aux conflits de droit issus de contrats individuels de travail (l'auteur indique qu'il a dû puiser grandement, pour ce qui est des données statistiques, dans l'ouvrage de McPherson et Meyers sur le sujet, qui date déjà de quelques années), analyse qui n'est aucunement aux dépens de celle, au besoin fortement critique, des procédés d'intervention dans les conflits collectifs.

\section{Pierre VERGE}

Disputes Procedure in Action, by Richard Hyman, London, Warwick Studies in Industrial Relations, Heineman Educational Books Ltd., 1972, 150 pp.

Cette monographie publiée par l'Industrial Relations Research Unit at Warwick consiste en une étude de la procédure de règlement des conflits en vigueur dans l'industrie de l'ingénierie (terme maintenant accepté comme traduction pour le terme anglais «engineering 》) de la région de Coventry en Angleterre.

L'ouvrage du Dr Hyman présente plus d'un intérêt pour le lecteur qui est plus ou moins au courant du fonctionnement $\mathrm{du}$ système de relations industrielles britannique. Disons d'abord que la procédure de règlement des conflits dans l'industrie de l'ingénierie est la plus ancienne et sans doute la plus importante de toutes les industries britanniques. Elle remonte à 1898 et, jusqu'à décembre 1971 où elle fut abandonnée définitivement, elle n'avait pas subi d'altérations fondamentales.

Cette procédure est sans doute le symbole le plus représentatif de toute 
l'économie traditionnelle des relations industrielles en Grande-Bretagne (jusqu'au pasage de l'Industrial Relations Act de 1971) en ce sens qu'elle repose essentiellement sur le volontarisme. Son fonctionnement était assez simple et il reposait essentiellement sur les éléments suivants. 1. Il s'agit d'une procédure qui s'adressait autant aux conflits d'intérêts qu'aux conflits de droits. 2. Comme les conventions collectives n'étaient pas de durée limitée comme en Amérique du Nord, les parties pouvaient en tout temps utiliser la procédure pour régler les problèmes issus de la situation de travail. 3. La mise en branle de la procédure ressemblait à la façon avec laquelle les griefs des conventions collectives nord-américaines sont initiés: on tentait de régler le problème d'abord au niveau de l'établissement par l'entremise d'une «Works Conference». Si le problème n'était pas réglé à ce niveau, il était reféré à la «Local Conference» groupant des représentants d'autres employeurs et autres syndicats de la région. Enfin, en cas d'échec à ce second niveau, le problème était soumis à la «Central Conference » composée de représentants d'associations nationales qui se réunissaient une fois par mois. Il est intéressant de noter que contrairement à la pratique nord-américaine, l'arbitre ( chairman $»)$ à chaque niveau était un employeur. Ceci peut paraître contraire aux règles de la neutralité mais, en pratique, la procédure permettait véritablement aux travailleurs d'obtenir justice car les employeurs n'avaient jamais intérêt à laisser accumuler les frustations. D'ailleurs, jusqu'à la fin des années soixante, les syndicats s'étaient fort bien accommodés de la procédure. Une règle importante qui permettait à la procédure d'être efficace était l'acceptation par les parties qu'aucune décision ou compromis survenu entre les syndicats et les employeurs ne devait être utilisé comme précédents pour des cas ultérieurs.

L'analyse de Hyman (qui avait été faite quelques mois avant que la procédure ne soit abandonnée) permet de dégager d'une façon très judicieuse les facteurs qui ont contribué à son abandon.

Suite à une étude de cas détaillée, $\mathrm{Hy}$ man découvrit que la majorité des problèmes discutés lorsque la procédure était initiée relevait du domaine des conflits d'intérêts plutôt que des conflits de droits; plus particulièrement, ce sont des questions salariales qui étaient généralement soulevées. Hyman en conclut que la procédure en venait finalement à jouer le même rôle que la négociation collective en Amérique du Nord, d'autant plus que le règlement des conflits en faveur de l'une ou l'autre partie était largement influencée par leur pouvoir économique respectif.

Il semble que cette constatation de Hyman soit la plus pertinente de tout le volume. Lorsqu'on connaît les structures de négociation britannique traditionnelles qui s'appliquent à toute une branche d'activités ( $\ll$ industry-wide bargaining ») plutôt qu'à des entreprises particulières, il est tout à fait prévisible qu'à un moment donné des phénomènes de glissement de salaires («wage drift ») apparaissent. C'est donc par le biais de la procédure de règlement des conflits que les travailleurs au niveau des entreprises peuvent tirer profit de leur pouvoir de négociation vis-à-vis leurs employeurs. Trois facteurs bien précis viennent illustrer ce phénomène. D'abord, l'étude de Hyman révèle que depuis le milieu des années soixante la procédure de règlement des conflits est de plus en plus inefficace, i.e. les problèmes sont de moins en moins résolus au niveau de l'établissement ou au niveau local mais rebondissent plutôt au niveau de la «Central Conference 》. Ce qui arrive en pratique est ceci : les employeurs ne voulant pas faire de concessions au niveau de l'établissement, le problème est reféré aux échelons supérieurs. Comme au niveau national il n'y a pas de représentants des parties impliquées directement (c'est la règle), les problèmes sont examinés par des experts peu familiers avec la relation de pouvoir au niveau de l'établissement. La conséquence de cette situation amène le second facteur: les travailleurs au niveau de l'établissement qui ont un pouvoir économique déclenchent des grèves sauvages (« unconstitutional strikes 》) quand toutes les étapes de la procédure ont été expirées. Enfin, un dernier indice de l'effritement de l'efficacité de la procédure est la nature des problèmes réglés de façon informelle. Alors que le but même de la procédure est de favoriser les règlements informels 
sur les questions fondamentales, on constate que les seules questions qui sont maintenant réglées de cette façon sont des problèmes à caractère nonéconomique. Quant aux différends au sujet des salaires et conditions de travail, ils aboutissent presque toujours dans le formalisme de la procédure pour se terminer très souvent par des grèves sauvages.

Nous savons maintenant que ce sont ces grèves sauvages (non officiellement approuvées par les dirigeants syndicaux) qui ont alarmé l'opinion publique britannique et le gouvernement et qui ont amené le parlement à passer l'Industrial Relations Act. Cependant, l'étude de $\mathrm{Hy}$ man aura permis, même si ce n'était pas son objet premier, de placer ces grèves sauvages dans leur véritable contexte: ils sont la conséquence logique d'un système de relations industrielles vétuste où les parties immédiatement concernées ont de moins en moins un droit de regard sur leurs conditions de travail. Comme celui-ci le mentionne p. 66 * The use of sanctions may be intimately related to collective bargaining as such. The nature of contemporary industrial relations in engineering ensures that most references to procedure are merely sectional pay claims which happen not to have been resolved domestically. Whatever the perspicacity and bargaining skill of external negotiators, the eventual settlement is normally shaped largely by the relative strength of the domestic protagonists and the assessment each makes of the other's determination. Sanctions applied by the party seeking substantive change may provide a show of strength or an indication of determination and thus function as an integral part of the bargaining process. In other words, the use of procedure and strikes in breach of procedure may be complementary rather than contradictory activities 》.

Les syndicats se sont retirés de cette procédure en décembre 1971 en invoquant trois raisons fondamentales: les délais, les prérogatives des employeurs qui font de la procédure «un appareil de conciliation par l'employeur» et son inefficacité en général. Le gouvernement britannique a d'ailleurs lui aussi reconnu l'inefficacité de cette procédure de règlement des conflits et c'est pourquoi les réformes contenues dans l'Industrial Relations Act reconnaissent, entre autres choses, la nécessité de décentraliser le processus de négociation sur la base de ce qui se pratique en Amérique du Nord.

On peut signaler en terminant que le cadre d'analyse utilisé par Hyman est celui de l'approche "behavioraliste» de la négociation collective développée par des spécialistes comme Walton et McKersie, Ann Douglas et d'autres.

\section{Jean BOIVIN}

\section{Programmes Learning Aid for Personnel} Administration, by George S. Odiorne, Illinois, Homewood, Learning Systems Company, 1973, 122 pp.

Ce nouveau livre de Odiorne donne une synthèse du vaste domaine de l'administration du personnel, en s'inspirant d'un livre antérieur du même auteur (Personnel Administration by Objective, 1961). L'approche systémique facilite la compréhension des divers sujets traités.

Ce texte programmé et les 125 questions à la fin de l'ouvrage faciliteront l'acquisition des connaissances chez le lecteur. De plus, un glossaire définissant les principaux termes et les index (alphabétique mots et par sujets) sont utiles. Certains aspects sont bien traités, tels politiques du personnel, les communications, la discipline, la participation, tandis que d'autres apparaissent trop succincts : l'entraînement et le développement, la rémunération, etc.

Bref, ce livre sera utile pour les étudiants et les praticiens en administration du personnel, et aidera tout administrateur à prendre connaisance des nouvelles tendances en gestion des ressources humaines.

\section{Jean LADOUCEUR}

Effective Management, Social Psychological Approach, by David J. Lawless, New Jersey, Prentice-Hall Inc., Englewood Cliffs, 1972, 422 pp.

The main objective of this book is to bring behavioral research findings to practicing managers. It covers many areas of individual and group behavior, 